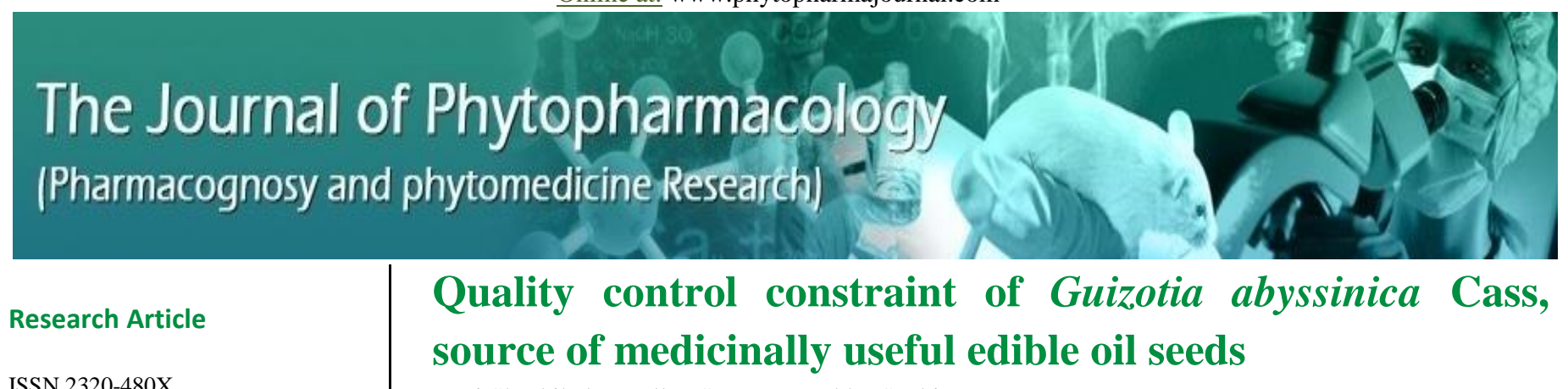

JPHYTO 2018; 7(5): 431-436

September- October

Received: 10-08-2018

Accepted: 17-09-2018

(C) 2018, All rights reserved

Bani Shashikala

PhD Scholar, Sri Dharmasthala

Manjunatheshwara (SDM) College of

Ayurveda, Kuthpady, Udupi, Karnataka,

India

Mallya Suma V

Associate Professor, Sri Dharmasthala Manjunatheshwara (SDM) College of Ayurveda, Kuthpady, Udupi, Karnataka, India

\section{Prabhu Suchitra}

Research Officer, Sri Dharmasthala Manjunatheshwara (SDM) College of Ayurveda, Kuthpady, Udupi, Karnataka, India
Correspondence:

Bani Shashikala

PhD Scholar, Sri Dharmasthala Manjunatheshwara (SDM) College of Ayurveda, Kuthpady, Udupi, Karnataka, India

Email: sumamallya@gmail.com
Bani Shashikala, Mallya Suma V, Prabhu Suchitra

\section{ABSTRACT}

Introduction: Guizotia abyssinica Cass. branched herb with erect, stout stem, commonly known as Ramtil or Niger seed. Seeds are the rich source of oil with high nutritional index. Traditionally the seed powder is used as remedy for cough, oil in cases of rheumatism. Apart from this the seeds are also used for different dishes like chutney, condiments etc. Hence a study has been designed to document pharmacognostic, physicochemical \& phytochemical data on above drug. Materials and Methods: Matured seeds were collected, authenticated and used for the study. Macro-microscopy, physicochemical, phytochemical standards and HPTLC marked using proper protocol. Results: Seeds are achene, obovoid and narrowly long like a needle, black with white to yellow scars on the top. Microscopic study shown the presence of epidermis and endodermis filled with alluerone grains and oil globules. Physico-chemical standards mark the purity of the drug. Alkaloid, Steroid, Carbohydrate, Terpenoid \& Coumarins were detected as secondary metabolites. HPTLC revealed prominent spots with Rf values $0.54,0.58$, \& 0.70 under short UV and $0.45 \& 0.82$ under long UV. Conclusion: Pharmacognostic values depicted in this paper are a measure of its quality standards.

Keywords: Guizotia abyssinica Cass, Ramtil, Pharmacognostic, Phytochemical, HPTLC

\section{INTRODUCTION}

'Guizotia abyssinica Cass.' from Compositae commonly known as 'Ramtil', 'Niger seed' is widely growing plant in Deccan provinces of India ${ }^{[1]}$. Being a native of Ethiopia, oil extracted from this seed is preferred as cooking oil ${ }^{[2]}$. The black oil seeds of the plant are traditionally used in houses of South India like Karnataka, Maharashtra, Andhra Pradesh up to Madhya Pradesh to make dry chutney, as a source of edible oil and used as an accompaniment with other food articles ${ }^{[3]}$. The seed powder is used as remedy for cough, oil in cases of rheumatism ${ }^{[4]}$.

The seeds are reported to contain nutritional components as oil $30-40 \%$, protein factor $10-25 \%$, sugar $12-18 \%$, fibre $10-20 \%$ and moisture content $10-11 \%$. The fixed oil present in the seed is said to be a mixture of triglycerides, palmitic, lauric, stearic acids ${ }^{[5]}$. Unsaponifiable matter is said to be mixture of stigmasterol and lupeol. The cold pressed oil is also used as substitute for sunflower oil/ olive oil ${ }^{[6]}$. Besides cookery, seed oil also said to be having utility in the preparation of soap, paint and other lubricants. The protein-rich seed content after oil extraction is said to be used as cattle feed, manure or fuel ${ }^{[7]}$.

Traditional claim suggests it as, healthy oil source, used in hot/dry climatic regions possessing rich nutritional value. Though widely used as a common food articles not any quality standards and activity profile have been investigated till today, hence an attempt to depict all in this paper.

\section{MATERIALS AND METHODS}

\section{Materials}

Matured seeds of Guizotia abyssinica Cass. (Ramtil) were collected from Gadag district, cleaned properly with other foreign matters like stem pieces, husks, taxonomically named, using floras,sample deposited at SDM centre for Research in Ayurveda and Allied sciences,(Voucher specimen No. 17032101).

\section{Macroscopy}

Outer features of samples were documented using Canon IXUS digital camera. The Macroscopic features were studied using taxonomy text books. Organoleptic characters marked as per standard guidelines ${ }^{[1]}$. 


\section{Microscopy}

Plant samples soaked in FAA (Formalin-5ml + Acetic acid-5ml + $70 \%$ Ethyl alcohol-90ml) solution for more than 48 hours. The seed samples were cut into thin transverse section using a blade and sections were stained with saffranine. Then these were mounted on slides visualized under microscope, photographed using Zeiss AXIO trinocular microscope attached with Zeiss AxioCam camera under bright field light. Magnifications of the figures are indicated by the scale-bars ${ }^{[2]}$.

\section{Powder microscopy}

One gram of test drug powder was sieved through 80 pore size mesh A pinch of powder was mounted on a slide with a drop of glycerine water. Slide visualized under microscope, particular cell features marked and recorded.

\section{Physicochemical study:}

Seed sample shade dried, powdered properly and loss on drying at $105^{\circ} \mathrm{C}$, total Ash, acid insoluble ash, water soluble ash, alcohol soluble extractive \& water-soluble extractive were carried out as per standard methodology ${ }^{[3]}$.

\section{Phytochemical study}

Alcoholic extract of seed powder was screened for the presence of various phytochemical constituents as per standard operative procedures and results recorded ${ }^{[4]}$.

\section{HPTLC}

$1 \mathrm{~g}$ of Guizotia abyssinica seed powder was extracted with $10 \mathrm{ml}$ of alcohol. 4,8 and $12 \mu \mathrm{l}$ of the above extract applied on a pre-coated silica gel $\mathrm{F}_{254}$ on aluminum plates to a band width of $7 \mathrm{~mm}$ using Linomat 5 TLC applicator. This particular plate was developed in Toluene: Ethyl acetate (9.0: 1.0). Then developed plates were visualized in short UV, long UV, and then derivatised with vanillin sulphuric acid observed under white light and scanned under UV $254 \mathrm{~nm}, 366 \mathrm{~nm}$ and $620 \mathrm{~nm}$. $R_{\mathrm{f}}$, colour of the spots and densitometric scan of this experiment were recorded.

\section{RESULTS}

\section{Macroscopy}

Guizotia abyssinica Cass. seed is an achene, club-shaped, obovoid and narrowly long like a needle, whitish yellow mark on the top as well as base with a hard testa. It appears glossy and shiny with characteristic odour. The embryo is white. (Figure $1 \&$ Table 1 ).

Table 1: Morphological characteristics of seeds of Guizotia abyssinica Cass.

\begin{tabular}{|c|c|c|}
\hline Sr. no & Parameters & Characters observed \\
\hline 1. & Colour & Light brown to black \\
\hline 2. & Shape & Club shaped, obovoid, needle like \\
\hline 3. & Appearance & Glossy \& shining \\
\hline 4. & Seed weight & $2.52 \mathrm{mg}$ \\
\hline
\end{tabular}

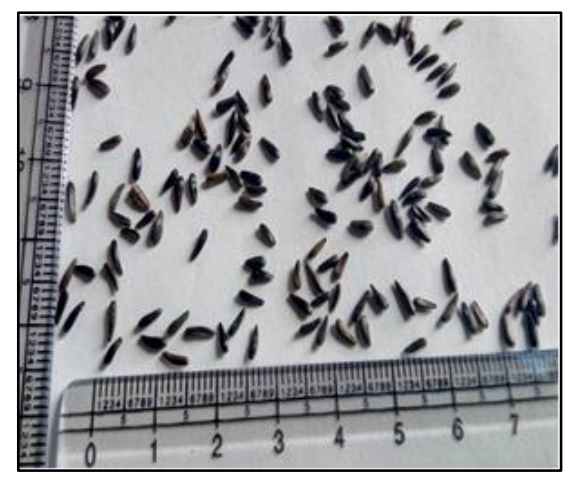

Fig 1: Macroscopy of Guizotia abyssinica Cass.

\section{Microscopy}

TS of seed show a distinct testa, thin endosperm and bulky cotyledons. Testa is differentiated clearly as outer and inner integuments. Outer integument shows well developed epidermis but thin, shining, single layered polygonal and tabular cells with full of mucilage. Sub-epidermis is 2 to 3 layered, brown with thick walled parenchyma (collenchyma).

Inner integument comprises of Sclerenchymatous layer reddish brown, single layered, longitudinally or radially elongated, compactly arranged with lignified stone cells. The cells beside being thick walled are pitted and show a very small lumen. Parenchymatous showed tangentially elongated, collapsed parenchymatous cells of one or two layers. Pigment layer: dark brown or chocolate brown, single layered, thick walled, with pitted rectangular cells.

Endosperm was narrow, thin which surround the cotyledons. The endosperm and cotyledons show parenchymatous, colourless, polyhedral, somewhat thick-walled cells containing aleurone grains and abundant oil globules. (Figure 2).

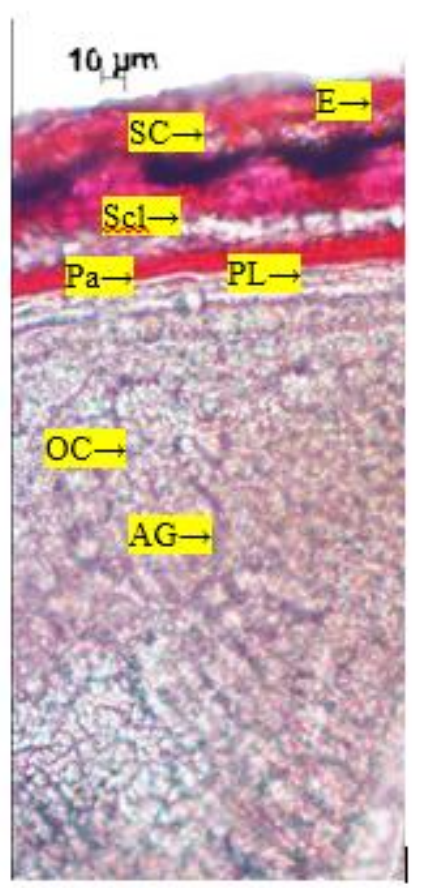

Figure 2: TS of Guizotia abyssinica Cass. 


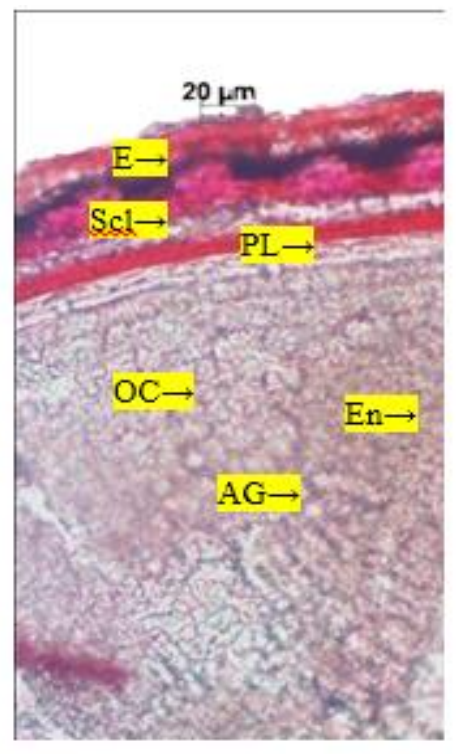

Fig 2a. TS of seed Guizotia abyssinica Cass.

AG - aleurone grains; Cot - cotyledon; E - epidermis; En - endosperm; OCOil cells; $\mathrm{Pa}$ - lignified parenchyma; $\mathrm{PL}$ - pigment layer; $\mathrm{SC}$ - stone cells; $\mathrm{Scl}$ - sclereids; $\mathrm{T}$ - testa.

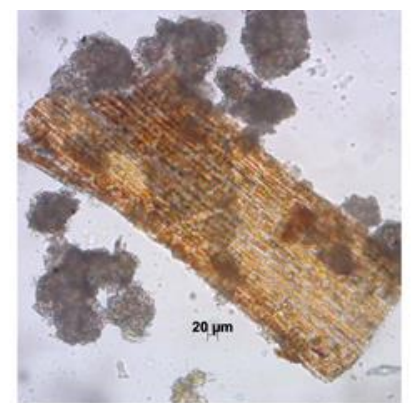

Fig. 3a. Sclerenchyma fibres

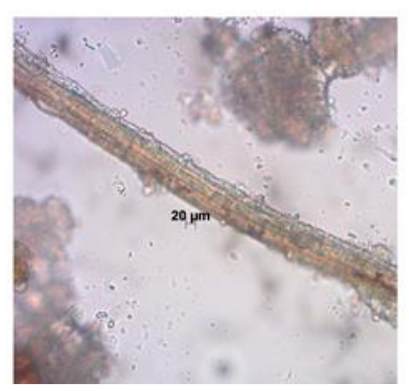

Fig. 3c. Bundle of fibres

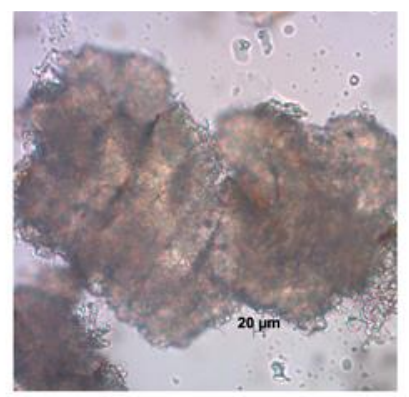

Fig 3e. Stonecells compactly arranged

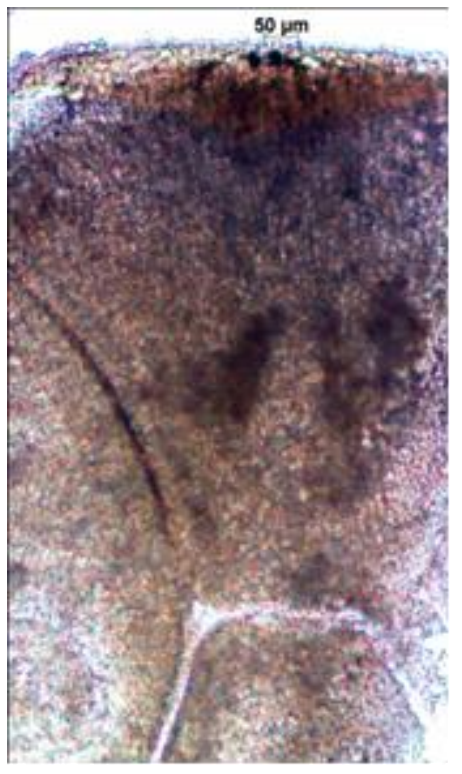

Fig 2c. T.S of seed of Guizotia abyssinica enlarged view without staining

\section{Powder microscopy}

Seed powder has shown following characters such as sclerenchyma fibres, bundle of fibres, sclerenchyma with pigments, stone cells and pitted sclerenchyma cells. (Figure 3)

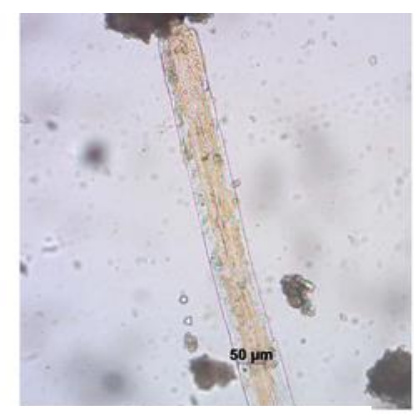

Fig. 3b. Fibre

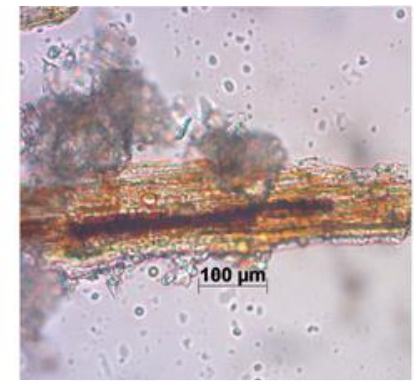

Fig 3d. Sclerenchyma with pigment layer

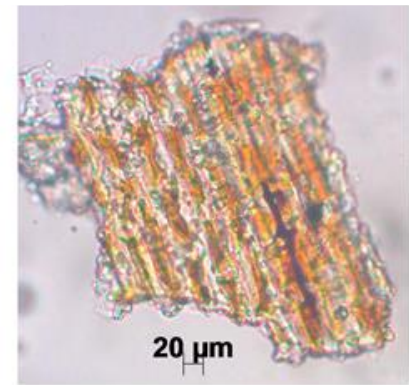

Fig 3f. Sclerenchyma pitted 


\section{Physico-chemical standards}

Loss on drying was $8.42 \%$, total ash $3.98 \%$, acid insoluble ash $0.2 \%$, water soluble ash $0.60 \%$, alchohol soluble extractive $26.94 \%$ and water-soluble extractive was $9.4 \% \mathrm{w} / \mathrm{w}$ respectively. (Table 2)

Table 2: Physico-chemical standards of Guizotia abyssinica Cass.

\begin{tabular}{|c|c|}
\hline Parameter & Results $\mathbf{n = 3}$ \% w/w \\
\hline Loss on drying & 8.42 \\
\hline Total Ash & 3.98 \\
\hline Acid Insoluble Ash & 0.2 \\
\hline Water soluble Ash & 0.60 \\
\hline Alcohol soluble extractive value & 26.94 \\
\hline Water soluble extractive value & 9.4 \\
\hline
\end{tabular}

\section{Phytochemical study}

Alchoholic extract of the test drug has shown the presence of Alkaloid, Steroid, Carbohydrate, Terpenoid \& Coumarins. (Table 3)

Table 3: Phytochemical constituents of Guizotia abyssinica Cass.

\begin{tabular}{|c|c|}
\hline Test & Inference \\
\hline Alkaloid & + \\
\hline Steroid & + \\
\hline Carbohydrate & - \\
\hline Tannin & - \\
\hline Flavanoids & - \\
\hline Saponins & + \\
\hline Terpenoid & + \\
\hline Coumarins & - \\
\hline Phenols & - \\
\hline Carboxylic acid & - \\
\hline Amino acids & + \\
\hline Resin & - \\
\hline Quinone & \\
\hline
\end{tabular}
(+) - present; (-) - negative

\section{HPTLC}

HPTLC of alcoholic extract revealed the prominent spots with $\mathrm{Rf}$ values $0.54,0.58, \& 0.70$ under short $\mathrm{UV}, 0.45 \& 0.82$ under long UV with selective mobile phase. (Figure 4,5 . Table 3 )

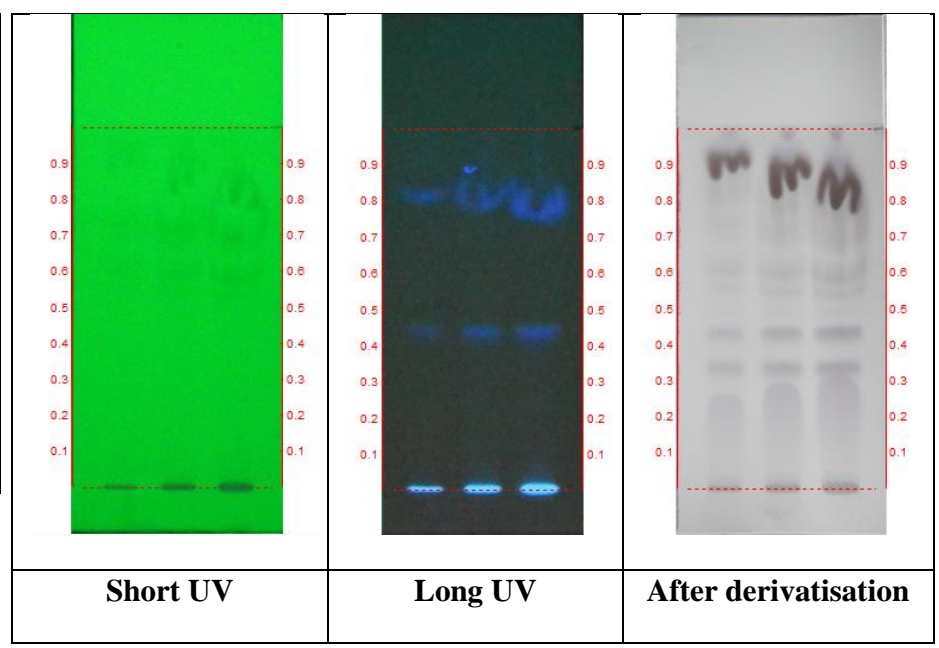

track 1- Guizotia abyssinica $-4 \mu 1$

track 2- Guizotia abyssinica $-8 \mu 1$

track 3- Guizotia abyssinica- $12 \mu 1$

\section{Solvent system - Toluene: Ethyl Acetate: (9:1)}

Figure 4. HPTLC photo documentation of ethanolic extract of Guizotia abyssinica Cass.

Table 3: $\mathrm{R}_{\mathrm{f}}$ values of samples

\begin{tabular}{|c|c|c|}
\hline Short UV & Long UV & Post derivatisation \\
\hline- & - & 0.34 (D. purple) \\
\hline- & 0.45 (F. blue) & 0.44 (D. purple) \\
\hline 0.54 (L. green) & - & 0.54 (L. purple) \\
\hline 0.58 (L. green) & - & - \\
\hline- & - & 0.60 (L. purple) \\
\hline- & - & 0.63 (L. purple) \\
\hline 0.70 (L. green) & - & 0.71 (L. purple) \\
\hline- & - & 0.75 (L. purple) \\
\hline - & - & 0.79 (L. purple) \\
\hline - & 0.82 (F. blue) & \multicolumn{1}{|c}{ - } \\
\hline *F- Fluorescent; L -Light; D - Dark
\end{tabular}




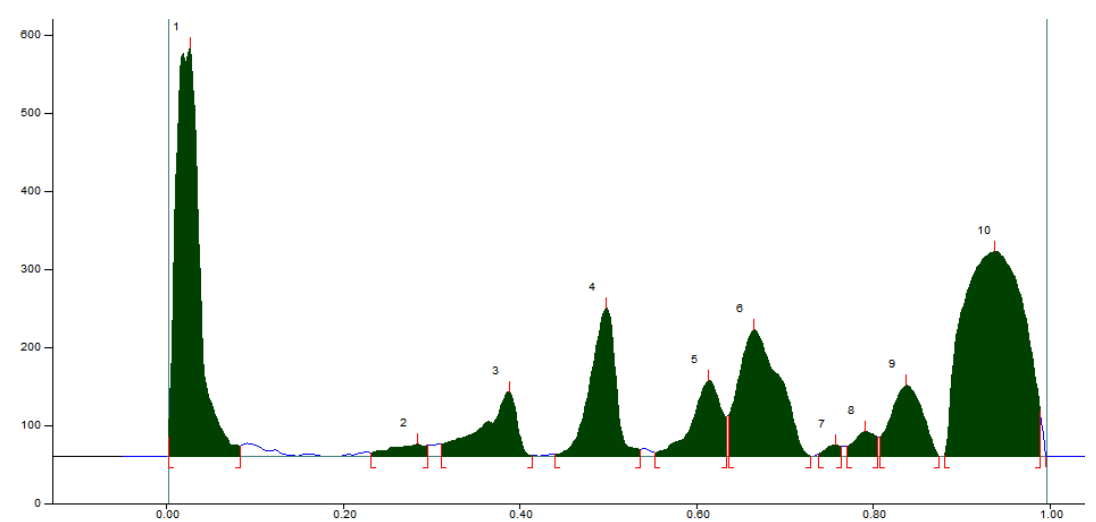

Track 3, ID: Guizotia abyssinica

\begin{tabular}{|c|c|c|c|c|c|c|c|c|c|}
\hline Peak & $\begin{array}{c}\text { Start } \\
\text { Position }\end{array}$ & $\begin{array}{c}\text { Start } \\
\text { Height }\end{array}$ & $\begin{array}{c}\text { Max } \\
\text { Position }\end{array}$ & $\begin{array}{c}\text { Max } \\
\text { Height }\end{array}$ & $\underset{\%}{\operatorname{Max}}$ & $\begin{array}{c}\text { End } \\
\text { Position }\end{array}$ & $\begin{array}{c}\text { End } \\
\text { Height }\end{array}$ & Area & $\begin{array}{c}\text { Area } \\
\%\end{array}$ \\
\hline 1 & $0.00 \mathrm{Rf}$ & $25.0 \mathrm{AU}$ & $0.03 \mathrm{Rf}$ & $522.3 \mathrm{AU}$ & $35.62 \%$ & $0.08 \mathrm{Rf}$ & $13.6 \mathrm{AU}$ & $10054.7 \mathrm{AU}$ & $24.86 \%$ \\
\hline 2 & $0.23 \mathrm{Rf}$ & $4.7 \mathrm{AU}$ & $0.29 \mathrm{Rf}$ & $15.5 \mathrm{AU}$ & $1.05 \%$ & $0.30 \mathrm{Rf}$ & $13.9 \mathrm{AU}$ & 452.2 AU & $1.12 \%$ \\
\hline 3 & $0.31 \mathrm{Rf}$ & $15.7 \mathrm{AU}$ & $0.39 \mathrm{Rf}$ & $82.6 \mathrm{AU}$ & $5.63 \%$ & $0.41 \mathrm{Rf}$ & 1.3 AU & $2284.8 \mathrm{AU}$ & $5.65 \%$ \\
\hline 4 & $0.44 \mathrm{Rf}$ & $2.3 \mathrm{AU}$ & $0.50 \mathrm{Rf}$ & $189.5 \mathrm{AU}$ & $12.93 \%$ & $0.54 \mathrm{Rf}$ & $9.2 \mathrm{AU}$ & $3860.3 \mathrm{AU}$ & $9.55 \%$ \\
\hline 5 & $0.55 \mathrm{Rf}$ & $4.8 \mathrm{AU}$ & $0.61 \mathrm{Rf}$ & $96.8 \mathrm{AU}$ & $6.60 \%$ & $0.64 \mathrm{Rf}$ & $50.8 \mathrm{AU}$ & $2304.6 \mathrm{AU}$ & $5.70 \%$ \\
\hline 6 & $0.64 \mathrm{Rf}$ & $52.1 \mathrm{AU}$ & $0.67 \mathrm{Rf}$ & 161.4 AU & $11.01 \%$ & $0.73 \mathrm{Rf}$ & $0.0 \mathrm{AU}$ & $5323.1 \mathrm{AU}$ & $13.16 \%$ \\
\hline 7 & $0.74 \mathrm{Rf}$ & $2.9 \mathrm{AU}$ & $0.76 \mathrm{Rf}$ & $14.4 \mathrm{AU}$ & $0.98 \%$ & $0.76 \mathrm{Rf}$ & $13.4 \mathrm{AU}$ & $177.0 \mathrm{AU}$ & $0.44 \%$ \\
\hline 8 & $0.77 \mathrm{Rf}$ & $12.8 \mathrm{AU}$ & $0.79 \mathrm{Rf}$ & $31.8 \mathrm{AU}$ & $2.17 \%$ & $0.81 \mathrm{Rf}$ & & $567.5 \mathrm{AU}$ & $1.40 \%$ \\
\hline 9 & $0.81 \mathrm{Rf}$ & $25.0 \mathrm{AU}$ & $0.84 \mathrm{Rf}$ & $90.4 \mathrm{AU}$ & $6.17 \%$ & $0.88 \mathrm{Rf}$ & $0.7 \mathrm{AU}$ & 2318.2 AU & $5.73 \%$ \\
\hline 10 & $0.88 \mathrm{Rf}$ & 1.1 AU & $0.94 \mathrm{Rf}$ & $261.5 \mathrm{AU}$ & $17.83 \%$ & $0.99 \mathrm{Rf}$ & $57.7 \mathrm{AU}$ & $13096.8 \mathrm{AU}$ & $32.39 \%$ \\
\hline
\end{tabular}

Fig 5a: At 254nm

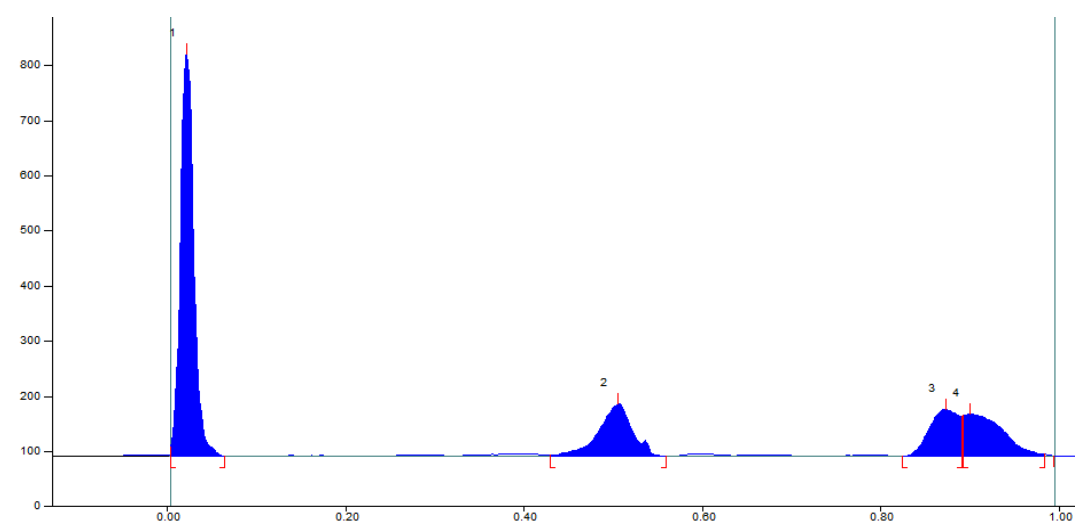

Track 3, ID: Guizotia abyssinica

\begin{tabular}{|c|c|c|c|c|c|c|c|c|c|}
\hline Peak & $\begin{array}{c}\text { Start } \\
\text { Position }\end{array}$ & $\begin{array}{c}\text { Start } \\
\text { Height }\end{array}$ & $\begin{array}{c}\text { Max } \\
\text { Position }\end{array}$ & $\begin{array}{c}\text { Max } \\
\text { Height }\end{array}$ & $\begin{array}{c}\text { Max } \\
\%\end{array}$ & $\begin{array}{c}\text { End } \\
\text { Position }\end{array}$ & $\begin{array}{c}\text { End } \\
\text { Height }\end{array}$ & Area & $\begin{array}{c}\text { Area } \\
\%\end{array}$ \\
\hline 1 & $0.00 \mathrm{Rf}$ & $16.8 \mathrm{AU}$ & $0.02 \mathrm{Rf}$ & $730.2 \mathrm{AU}$ & $74.08 \%$ & $0.06 \mathrm{Rf}$ & $0.0 \mathrm{AU}$ & $7883.9 \mathrm{AU}$ & $52.92 \%$ \\
\hline 2 & $0.43 \mathrm{Rf}$ & $1.6 \mathrm{AU}$ & $0.51 \mathrm{Rf}$ & $94.7 \mathrm{AU}$ & $9.60 \%$ & $0.56 \mathrm{Rf}$ & $0.1 \mathrm{AU}$ & $2556.5 \mathrm{AU}$ & $17.16 \%$ \\
\hline 3 & $0.83 \mathrm{Rf}$ & $0.1 \mathrm{AU}$ & $0.87 \mathrm{Rf}$ & 84.7 AU & $8.59 \%$ & $0.89 \mathrm{Rf}$ & $73.0 \mathrm{AU}$ & $2026.8 \mathrm{AU}$ & $13.60 \%$ \\
\hline 4 & $0.89 \mathrm{Rf}$ & $73.4 \mathrm{AU}$ & $0.90 \mathrm{Rf}$ & $76.2 \mathrm{AU}$ & $7.73 \%$ & $0.99 \mathrm{Rf}$ & $3.3 \mathrm{AU}$ & $2431.3 \mathrm{AU}$ & $16.32 \%$ \\
\hline
\end{tabular}

Fig 5b: At 366nm 


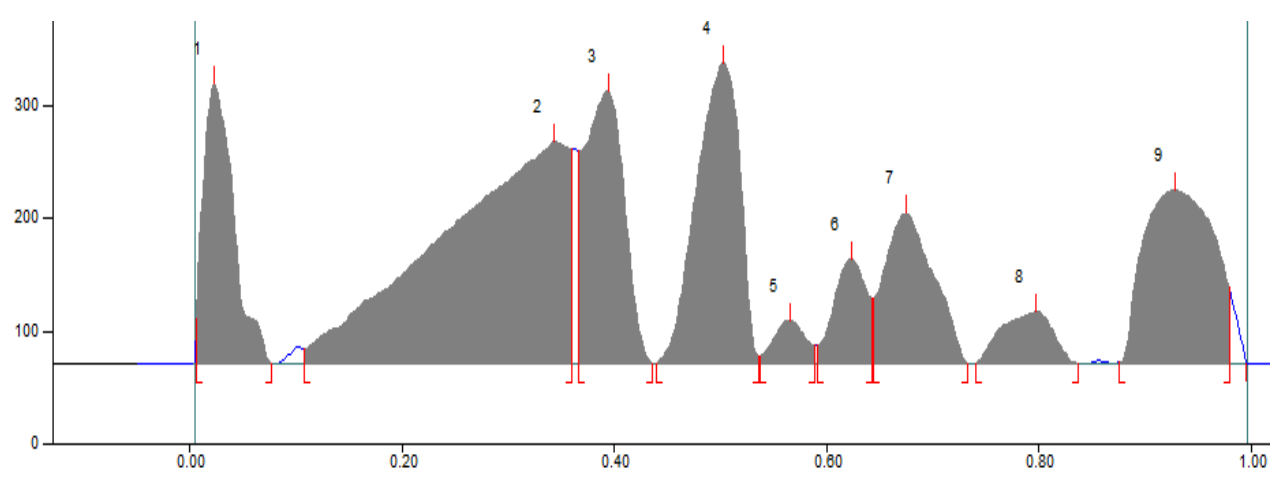

Track 3, ID: Guizotia abyssinica

\begin{tabular}{|c|c|c|c|c|c|c|c|c|c|}
\hline Peak & \begin{tabular}{|c|} 
Start \\
Position
\end{tabular} & $\begin{array}{c}\text { Start } \\
\text { Height }\end{array}$ & \begin{tabular}{c|} 
Max \\
Position
\end{tabular} & $\begin{array}{c}\text { Max } \\
\text { Height }\end{array}$ & $\underset{\%}{\operatorname{Max}}$ & \begin{tabular}{|c|} 
End \\
Position
\end{tabular} & \begin{tabular}{|l|} 
End \\
Height
\end{tabular} & Area & $\begin{array}{c}\text { Area } \\
\%\end{array}$ \\
\hline 1 & $0.01 \mathrm{Rf}$ & $39.2 \mathrm{AU}$ & $0.02 \mathrm{Rf}$ & $247.2 \mathrm{AU}$ & $17.46 \%$ & $0.08 \mathrm{Rf}$ & $0.1 \mathrm{AU}$ & 5147.9 AU & $9.72 \%$ \\
\hline 2 & $0.11 \mathrm{Rf}$ & $13.0 \mathrm{AU}$ & $0.34 \mathrm{Rf}$ & $196.9 \mathrm{AU}$ & $13.91 \%$ & $0.36 \mathrm{Rf}$ & $89.3 \mathrm{AU}$ & 17149.7 AU & $32.38 \%$ \\
\hline 3 & $0.37 \mathrm{Rf}$ & $188.9 \mathrm{AU}$ & $0.40 \mathrm{Rf}$ & $241.5 \mathrm{AU}$ & $17.06 \%$ & $0.44 \mathrm{Rf}$ & $0.2 \mathrm{AU}$ & $6512.8 \mathrm{AU}$ & $12.30 \%$ \\
\hline 4 & $0.44 \mathrm{Rf}$ & $0.4 \mathrm{AU}$ & $0.50 \mathrm{Rf}$ & $265.8 \mathrm{AU}$ & $18.77 \%$ & $0.54 \mathrm{Rf}$ & $6.6 \mathrm{AU}$ & $7857.9 \mathrm{AU}$ & $14.84 \%$ \\
\hline 5 & $0.54 \mathrm{Rf}$ & $6.6 \mathrm{AU}$ & $0.57 \mathrm{Rf}$ & $38.2 \mathrm{AU}$ & $2.70 \%$ & $0.59 \mathrm{Rf}$ & $16.0 \mathrm{AU}$ & $826.0 \mathrm{AU}$ & $1.56 \%$ \\
\hline 6 & $0.59 \mathrm{Rf}$ & $16.1 \mathrm{AU}$ & $0.62 \mathrm{Rf}$ & $92.9 \mathrm{AU}$ & $6.56 \%$ & $0.64 \mathrm{Rf}$ & $58.0 \mathrm{AU}$ & $2043.8 \mathrm{AU}$ & $3.86 \%$ \\
\hline 7 & $0.65 \mathrm{Rf}$ & $58.1 \mathrm{AU}$ & $0.68 \mathrm{Rf}$ & $133.3 \mathrm{AU}$ & $9.41 \%$ & $0.73 \mathrm{Rf}$ & $0.4 \mathrm{AU}$ & $4468.8 \mathrm{AU}$ & $8.44 \%$ \\
\hline 8 & $0.74 \mathrm{Rf}$ & $0.2 \mathrm{AU}$ & $0.80 \mathrm{Rf}$ & $46.0 \mathrm{AU}$ & $3.25 \%$ & $0.84 \mathrm{Rf}$ & $0.1 \mathrm{AU}$ & $1607.9 \mathrm{AU}$ & $3.04 \%$ \\
\hline 9 & $0.88 \mathrm{Rf}$ & $1.4 \mathrm{AU}$ & $0.93 \mathrm{Rf}$ & $153.9 \mathrm{AU}$ & $10.87 \%$ & $0.98 \mathrm{Rf}$ & $67.4 \mathrm{AU}$ & $7347.2 \mathrm{AU}$ & $13.87 \%$ \\
\hline
\end{tabular}

Fig 5c: At $620 \mathrm{~nm}$

\section{DISCUSSION}

Guizotia abyssinica Cass the source of oil seeds, which grows in drier regions of India, and possessing many precious nutrients. The cold pressed oil is also used as substitute for different cooking oils. Wide therapeutic and edible utility of this less studied seed oil is need of an hour to document pharmacognostic standards.

Guizotia abyssinica Cass. is an achene, of club-shaped, obovoid and narrowly long like a needle, black with white to yellow scars on the top. On naked eye observation the seeds appear glossy and shiny with characteristic odour. Transverse section of the seed revealed two integuments, outer \& inner. Outer integument consists of well developed, thin, shining, single layered polygonal and tabular celled epidermis with full of mucilage and 2 to 3 layered sub-epidermis, brown with thick walled parenchyma. Inner integument comprises of single layered sclerenchymatous layer reddish brown, with longitudinally or radially elongated, compactly arranged lignified stone cells. These cells beside being thick walled are pitted and show a very small lumen, collapsed parenchymatous cells \& dark brown or chocolate brown in colour. Thin endosperm which surrounds the cotyledons is the feature of TS. The cells of endosperm and cotyledons are parenchymatous, colorless, polyhedral, somewhat thick walled containing aleurone grains and abundant oil globules.

Physicochemical parameters of a drug indicate chemical nature, physical impurities due to contamination as well as the solubility in different extractive media. Alcohol soluble extractive value, represent their solubility in respective media along with chemical nature. $26.94 \% \mathrm{w} / \mathrm{w} \& 9.4 \% \mathrm{w} / \mathrm{w}$ were extractive values of test drug obtained in alcohol and water respectively, which show its solubility in alcoholic media. Test drug show the presence of carbohydrate, alkaloid, steroid, terpenoids, coumarins \& resin. HPTLC profile exposed the prominent spots with $\mathrm{Rf}$ values $0.54,0.58, \& 0.70$ under short UV, $0.45 \& 0.82$ under long UV with selective mobile phase.

\section{CONCLUSION}

Guizotia abyssinica. Cass is an edible oil seed with therapeutic utility, studied pharmacognostically. As authenticity is the first parameter in any drug research, results presented in this paper will be beneficial to carry out further researches.

\section{Acknowledgement}

Authors are obliged to President, Dr. D. Veerendra Heggade, SDM Educational Society for constant support in research activities. Authors are indebted to Dr. G Srinivasa Acharya, Principal SDM Udupi and Dr. B. Ravishankar, Former Research Director, SDM Centre for Research in Ayurveda and Allied Sciences, Udupi for Guidance and support.

\section{REFERENCES}

1. Kirtikar KR. \& Basu BD. Indian medicinal plants. volII, Dehradun; International Book distributors; 2006, P.1369

2. Mulata Gelata, Rodomiro Ortiz, The importance of Guizotia abyssinica (niger) for sustainable food security in Ethiopia: Genetic Resources and crop evolution June 2013; 60(5):1763-70

3. Anonymous. The wealth of India, A dictionary of Indian raw materials \& industrial products Vol-IV. Newdelhi: Council of scientific \& industrial research: 2009, P.270- 275

4. Khare CP, Indian medicinal Plants an illustrated dictionary, Springer: 2007, P. 297

5. Nadakarni KM., Indian Material Medica Vol-I, Dehradun; International book Distributor: 2006, P.395

6. Dwivedi Sumeet, and Kohli Seema Folk-lore uses of Guizotia abyssinica (L.F) Cass. Among tribal and rural people of Madhya Pradesh, IJPTP 2012; 3(4):434-437.

7. Wallis TE. Textbook of Pharmacognosy. New Delhi: CBS Publisher and Distributors: $1985 ;$ P.527

\section{HOW TO CITE THIS ARTICLE}

Shashikala B, Mallya Suma V, Suchitra P. Quality control constraint of Guizotia abyssinica Cass, source of medicinally useful edible oil seeds. J Phytopharmacol 2018; 7(5):431-436. 